\title{
Collapse of a new type of self-expanding metallic tracheal stent
}

\author{
R. Trisolini1, D. Paioli1, V. Fornario2, L. Lazzari Agli3, \\ D. Grosso4, M. Patelli1
}

ABSTRACT: Collapse of a new type of self-expanding metallic tracheal stent. R. Trisolini, D. Paioli, V. Fornario, L. Lazzari Agli, D. Grosso, M. Patelli.

Alveolus TB-STSTM is a new self-expanding, completely polyurethane-covered, metallic stent which has been designed to be successfully used even in the treatment of non-neoplastic airway strictures as it is supposed to be removable. We recently observed the collapse of an Alveolus tracheal stent, causing dyspnea and hemopthysis, in a 63-yr-old female patient with post-intubation tracheal stenosis. Such a complication, which to our knowledge has never been previously reported with metallic stent use, forced us to remove the stent.

Monaldi Arch Chest Dis 2006; 65: 1, 56-58.

Keywords: Tracheal stenosis, stent, bronchoscopy.

${ }^{1}$ Unit of Thoracic Endoscopy and Pulmonology, Maggiore Hospital, Bologna,

2 Biomedical Engineer, Private Advisor, Bologna,

3 Unit of Pulmonology, City Hospital, Rimini,

${ }^{4}$ Unit of Pulmonology, Bellaria Hospital, Bologna, Italy.

Correspondence: Marco Patelli, MD, FCCP, Unit of Thoracic Endoscopy and Pulmonology, Maggiore Hospital, Largo Nigrisoli 2, 40100 Bologna, Italy; e-mail: marco.patelli@ausl.bologna.it

\section{Introduction}

Airway stent insertion is a procedure increasingly used in patients with complex post-intubation tracheal stenosis (PITS) who are unfit for or refuse surgery [1-3]. Very recently, a new self-expanding metallic stent (Alveolus TB-STSTM, Alveolus Inc., Charlotte, NC, USA) which is supposed to be easy to remove and for which indication for the treatment of benign tracheobronchial conditions has been obtained from the FDA, has been put on the market and is under evaluation. We report on a very unusual complication of an Alveolus tracheal stent in a patient with PITS.

\section{Case report}

A 63-yr-old, non-smoking female patient was referred to our Thoracic Endoscopy Unit in September 2004 for evaluation of complex post-intubation tracheal stenosis (PITS) which had developed after prolonged invasive mechanical ventilation (1 month) following emergency surgery for acute dissection of the aorta. At flexible bronchoscopy examination, a severe (diameter $<6 \mathrm{~mm}$; length $>1$ ring) fibrous tracheal stenosis was seen at $6 \mathrm{~cm}$ below the vocal cords, in the area where the cuff of the tracheal tube had been positioned. The patient was submitted to Nd-YAG laser-assisted mechanical dilation during rigid bronchoscopy, with complete restoration of airway patency. One month later, however, she began complaining of exertional dyspnea and stridor. As recurrence of severe tracheal stenosis was seen at flexible bronchoscopy, Nd-YAG laser-assisted mechanical dila- tion during rigid bronchoscopy was again performed. After the procedure, however, the scarring area sharp-angled the airway lumen to the right. The patient was proposed the opportunity of evaluation for possible surgery, but she refused. One month later, as stridor developed and recurrence of severe tracheal stenosis was ascertained (figure 1a), a new Nd-YAG laser-assisted mechanical dilation under rigid bronchoscopy was performed with the aim of inserting a tracheal stent. Unfortunately, any attempt to go past the stenotic area with a tracheoscope $(14 \mathrm{~mm})$ in order to insert a hourglass silicone stent caused significant mucosal damage mainly due to the sharp angulation of the tracheal lumen, and was interrupted because of the risk of tracheal rupture. At this point, steps were taken to insert a 16 x $40 \mathrm{~mm}$ Alveolus TB-STSTM tracheal stent under direct vision. The stent opened easily, deployed fully (figure 1b), and completely bridged the stenotic area allowing patency of the airway and recovery of a near-straight axis of the tracheal lumen. After extubation, however, in the awakening phase, the patient abruptly developed hemopthysis and stridor after coughing. At bronchoscopy, collapse of the stent with relevant airway obstruction could be seen (figure 1c), and emergency removal with alligator forceps during rigid bronchoscopy was easily and successfully performed. At this point, the patient accepted evaluation for possible surgery.

\section{Discussion}

The best definitive treatment for patients with complex PITS is unanimously thought to be surgi- 


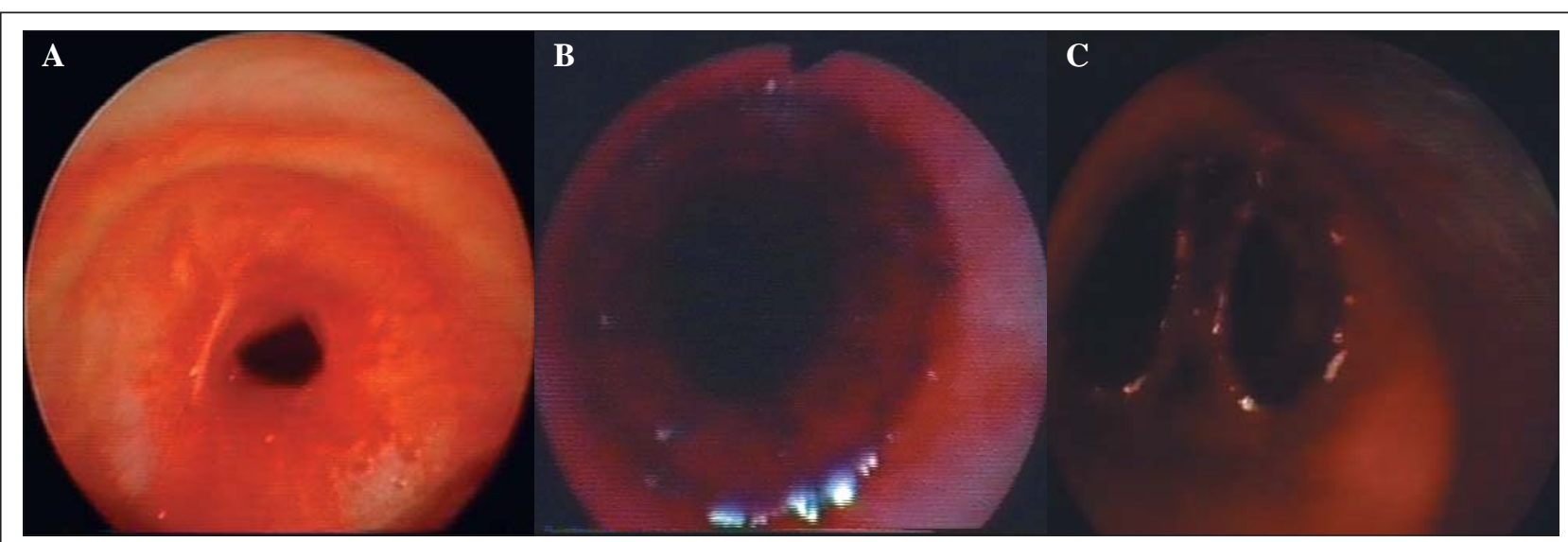

Fig. 1. - Endoscopic view of the mid-trachea:

a) Severe, post-intubation fibrous tracheal stenosis before endoscopic treatment;

b) after laser-assisted mechanical dilation, a fully deployed Alveolus TB-STSTM tracheal stent bridges the stenosis;

c) the Alveolus TB-STSTM tracheal stent has collapsed.

cal [3]. Nevertheless, for the increasing number of patients who are not considered candidates for major surgical intervention due to comorbid medical conditions (either temporarily or permanently) or who refuse surgery, endoscopic treatment is warranted [1, 2]. Balloon-bronchoplasty, as well as laser or electrocautery-assisted mechanical dilation with a rigid bronchoscope may be attempted as a first-step endoscopic treatment, but most patients with complex PITS will require a stent insertion [2, 4]. Several Authors have reported good results and acceptable complication rates with the use of silicone stents, which offer the chance of relatively easy removal if the patient becomes a secondary candidate to surgery, and are therefore rightly regarded as the "gold standard" prostheses in this setting $[1,2,5,6]$.

As compared to silicone stents, SEMS are less likely to migrate and can be placed under flexible bronchoscopy, but their use in benign stenoses has been limited by the fact that they are extremely difficult to remove and frequently undergo inner obstructive granulomata $[1,7,8]$. In a recent study, Gaissert et al. reported on a series of patients with difficult-to-solve complications by SEMS, which had been positioned for benign tracheobronchial strictures, and suggested that the current generation of SEMS should be avoided in benign strictures of trachea and bronchi [9].

Very recently, a new SEMS (Alveolus TBSTSTM, Alveolus Inc., Charlotte, NC, USA) with indication also for benign airway strictures has been put on the market and is under evaluation. This stent has been manufactured with the idea of preserving the advantages of metallic stents while limiting their main disadvantages, namely obstructive granulomata and difficult removal. The Alveolus TB-STSTM Stent, in fact, is completely covered with a biocompatible polyurethane membrane and the stent edges are slightly vaulted inwardly in order to limit airway injury; these characteristics are supposed to minimise the formation of obstructive granulomata and make stent removal possible. Furthermore, the stent is designed with a slightly larger diameter near the distal and proxi- mal ends in order to minimise the risk of migration.

In the case we are reporting, we had in mind the positioning of a hourglass silicone stent, but we did not succeed as the airway lumen in the stenotic area, even after dilation, sharp-angled the trachea making the passage of the tracheoscope impossible and dangerous. As the patient's PITS had already recurred twice after laser-assisted mechanical dilation and surgery was refused, we decided to insert an Alveolus TB-STSTM Stent, after considering its above-mentioned properties. The stent proved easy to position, deployed fully, and was perfectly patent when the patient was extubated, but it folded inwardly in the awakening phase while the patient was coughing. It is very difficult to determine which factor(s) caused such a complication, which to our knowledge has never been reported with other currently available SEMS. SEMS, in fact, exhibit the better internal:external diameter ratio, and are thought to be flexible enough to change shape during the stress of coughing, yet keeping an excellent radial strength during constant compression by stenosis $[1,10]$.

The possibility of a defective manufacture of the stent has to be considered. Interestingly, an FDA enforcement report dating back to October 6, 2004 signalled the risk of collapse of 47 Alveolus TB-STSTM stents of various size and lots, all of them distributed in the USA, due to incomplete polyurethane-coating. Unfortunately, in the rush of the emergency, the stent that we used was mistakenly thrown away after removal and couldn't be retrieved, so that we couldn't send it to the manufacturer for failure analysis.

As for the size, we do not believe that our choice had a role in this case as we followed the suggested indication for appropriate size selection provided by the manufacturer, as well as the experience with other currently available SEMS. As far as the length is concerned, the stent bridged the target stenosis with a margin $>5 \mathrm{~mm}$ both proximally and distally. The diameter, on the other hand, did not exceed by more than $2 \mathrm{~mm}$ the diameter of the normal proximal tracheal lumen. 
A final possible explanation for the reported complication, which will need a larger range of experience to be verified, includes a sub-optimal compatibility between the geometrical properties of the Alveolus TB-STSTM stent and specific airway conditions/structures.

In conclusion, we believe it is important for the community of interventional bronchoscopists to be aware of such a complication in order to strictly follow-up patients who are submitted to positioning of this new SEMS.

\section{References}

1. Ernst A, Feller-Kopman D, Becker HD, Mehta AC. Central airway obstruction. Am J Resp Crit Care Med 2004; 169: 1278-97.

2. Brichet A, Verkindre C, Dupont J, et al. Multidisciplinary approach to management of post-intubation tracheal stenoses. Eur Resp J 1999; 13: 888-93.

3. Grillo HC. Post-intubation tracheal stenosis. Treatment and results. J Thorac Cardiovasc Surg 1995; 109: 486-92.
4. Noppen M, Schlesser M, Meysman M, D'Haese J, Peche R, Vincken W. Bronchoscopic balloon dilatation in the combined management of postintubation stenosis of the trachea in adults. Chest 1997; 112: 1136-40.

5. Martinez-Ballarin JL, Diaz-Jimenez JP, Castro MJ, Moya JA. Silicone stents in the management of benign tracheobronchial stenoses. Tolerance and early results in 63 patients. Chest 1996; 109: 626-9.

6. Vergnon JM, Costes F, Polio JC. Efficacy and tolerance of a new silicone stent for the treatment of benign tracheal stenosis: preliminary results. Chest 2000; 118: 422-6.

7. Noppen M, Van Renterghem D, Vanderstraeten P. The wrong stent at the wrong time: a cautionary tale. Respiration 2003; 70: 313-6.

8. Saad CP, Murthy S, Krizmanich G, Mehta AC. Self-expandable metallic airway stents and flexible bronchoscopy. Chest 2003; 124: 993-9.

9. Gaissert HA, Grillo HC, Wright CD, et al. Complication of benign tracheobronchial strictures by self-expanding metal stents. J Thorac Cardiovasc Surg 2003; 126: 744-7.

10. Freitag L, Eicker K, Donovan TJ, Dimov D. Mechanical properties of airway stents. J Bronchol 1270-8.

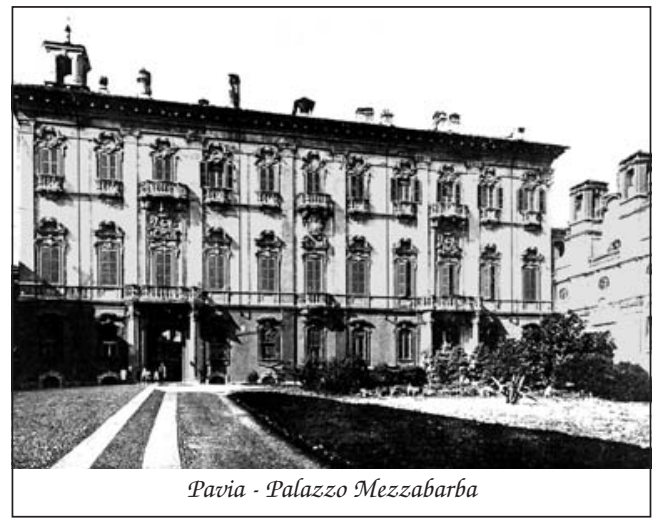

